\title{
Scattering of Electromagnetic Waves From Rough Surfaces: A Boundary Integral Method for Low-Grazing Angles
}

\author{
Philippe Spiga, Gabriel Soriano, and Marc Saillard
}

\begin{abstract}
We present a boundary integral method for the numerical solution of the rigorous problem of wave scattering from rough surfaces under grazing illumination. The model of a locally perturbated plane is adopted: a finite patch of rough surface has its roughness flattened at the edges. The boundary formulation unknowns are the tangential components of the scattered field, defined as the contribution from the rough area. This way, the numerical domain of study is correctly bounded, even with a plane wave as incident field, and the sampled area is made independent of the incidence. This rigorous approach, called the grazing method of moments, is implemented on two-dimensional perfectly conducting surfaces and validated by comparison with a reference numerical solution for surfaces with Gaussian correlation functions. Now, the validity of approximate models at low-grazing-angles can be investigated; the small perturbation method and the small slope approximation are addressed in this paper. Scattering diagrams show how the performances of these methods deteriorate drastically at backward scattering angles as the incidence goes to grazing.
\end{abstract}

Index Terms-Boundary integral equations, electromagnetic scattering by rough surfaces, low-grazing angles.

\section{INTRODUCTION}

$\mathbf{T}$ HE backscattering of electromagnetic waves from rough surfaces at low-grazing angle is a specific and difficult topic [1]. In particular, the usual criteria of validity of approximate methods have to be revisited. Indeed, depending on whether one focuses onto forward scattering or back scattering, the accuracy of approximate methods may differ drastically. The efficiency of shadowing functions on high frequency approximations is also difficult to estimate. Some specific methods have recently been proposed, both analytical [2], [3] and numerical [4], [5] but they address one-dimensional surfaces. At present, all published comparisons with rigorous methods at grazing appear to be restricted to one-dimensional surfaces. This proves that current numerical formulations feel uncomfortable at low-grazing angles. The aim of this paper thus is to propose a model for the scattering of electromagnetic

Manuscript received July 10, 2007; revised January 8, 2008. Published July 7, 2008 (projected).

P. Spiga is with the DCNS, 83000 Toulon, France and also with the Laboratoire de sondage electromagnetiques de l'environnement terrestre (LSEET), Fresnel Institute, 13397 Marseille Cedex 20, France.

G. Soriano is with Paul Cezanne University, 13628 Marseille Cedex 20, France and also with the ElectroMagnetical and Optical remote Sensing (SEMO) Team, Fresnel Institute, 13397 Marseille Cedex 20, France (e-mail: gabriel.soriano@fresnel.fr).

M. Saillard is with the Laboratoire de sondage electromagnetiques de l'environnement terrestre (LSEET), University of Toulon, 83957 La Garde, France.

Digital Object Identifier 10.1109/TAP.2008.924710 waves from two-dimensional surfaces, based on a boundary integral formalism, tailor-made for backscattering at low-grazing angles.

The direct problem of the scattering of electromagnetic waves from a random rough surface in harmonic regime can be rigorously solved only by means of numerical methods [6], [7]. When the surface separates two homogeneous media, the prime approach is the boundary integral formalism. The scattering problem is reduced to the search of the tangential components of the total electric and magnetic fields on the boundary. Integral equations are cast into a linear system using the method of moments (MoM) or the boundary element method. Sampling has to be made at the scale of the wavelength. In the physical problem, the incident field is a beam with a footprint on the surface at least several hundreds wavelengths long. This provides by far too many unknowns for numerical solution. However, it is now well established [7] that the scattered field can be estimated through ensemble average over smaller samples, with the corresponding lower angular resolution. Several models have been investigated for the scattering from those small rough surface patches. We now review them from a low-grazing angle point of view.

- First, an infinite rough surface is enlightened by a tapered beam, generally of Gaussian amplitude [8]-[11], with footprint of prescribed dimensions. As long as the short-coupling-range phenomenon can be invoked [12], the support of the surface unknowns is only some wavelengths larger than the footprint, and the domain of study is thus well bounded. This model is very close to the physical problem. However, with a tapered beam, there is a minimum surface length to consider, that increases asymptotically as the inverse of the squared grazing angle, see [13] for a discussion. The tapered beam model has been widely applied to the scattering from unidimensional surfaces, at grazing angles as low as $1^{\circ}$ [14]. The minimum grazing angle for two-dimensional surfaces is much higher.

- Second, the roughness is assumed to be periodic, and the incident field is a plane wave. This is a problem of scattering by a diffraction grating, that can be very efficiently solved by specific methods [15], [16]. However, for a given incidence, the scattered field is restricted to discrete directions. Also, grating methods show specific difficulties, such as the Rayleigh anomaly. Finally, natural surfaces are not periodic, and the influence of the periodic boundary conditions on the computed scattered field is difficult to estimate.

- In a third model, the surface is a bounded perturbation of the average plane under plane wave illumination. Here, some supplementary step is required to bound the domain 
of study. The electrical image theory can do the job in a very elegant way. It is however restricted to perfectly conducting surfaces, since the half-space Green's function is analytical only for this boundary condition. Another tapering method, inherited from resistive strips [17], [18], consists in adding resistive regions on the edges of the rough region. It has been succesfully applied at lowgrazing incident angles to the prediction of forward scattering [19]. However, for backscattering, resistive tapering is claimed in [20] to be restricted to grazing angles larger than $20^{\circ}$. This may result from a lack of accuracy of the method, since, at grazing incidence, only a very weak part of the incident field is backscattered.

This last model has been extensively studied for the Helmholtz equation under the finite section method designation. See [21] for a short review. In this same paper, stability of the bounded perturbation problem and its convergence toward the infinite rough surface problem as the dimensions of the perturbation grow has been rigorously proved. This is an important result, with no equivalent for periodic surface.

Therefore, let us reconsider the model of the bounded perturbation of a plane, without resistive tapering. Under plane wave illumination, reflection on the flat part is the main contribution to the scattering amplitude in the forward direction. Since we are concerned by the contribution from roughness, we define a scattered field that is free of the field that would be reflected from a plane interface and we suggest to choose the tangential electric and magnetic components of that scattered field as surface unknowns.

On one hand, this should provide better accuracy out of the specular reflection direction. On the other hand, surface unknowns should decrease away from the rough area, since their value result from interactions with the rough area or from the propagation of surface waves. Therefore, it is assumed that the support of the tangential components of the scattered field has similar dimensions as the rough region, say some wavelengths larger. The boundary integral formalism based on that choice of surface unknowns is developed in Section II, where we present integral equations and far-field formulas for these unknowns.

For a plane wave impinging a plane interface with bounded roughness, the behavior of the scattered field in the far-field when the grazing incidence angle or the grazing scattering angles tends toward zero can be theoretically predicted [22]. These results, that depend on the boundary condition, are summarized in the beginning of Section III. However, as outlined earlier, surface waves (surface plasmon polaritons SPP) may propagate, whatever the polarization on a two-dimensional rough surface. Even though they no longer contribute to the far field once propagating over the flat area, SPP are part of the interaction process, and as such, cannot be discarded in integral equations. Consequently, with a restricted domain of study, the SPP may be truncated and may radiate propagative waves around the grazing directions. To get rid of these artifacts, we propose in Section III modified scattering formulas that enforce the theoretical behavior of the scattered field at grazing. These formulas are directly inspired from the work by Tatarskii and Charnotskii [22].

Next, this approach, called the grazing MoM, is applied to perfectly conducting surfaces. Details on numerical implementation are given in Section IV. Validation by comparison with a classical numerical method, at non-grazing incidence, follows in Section V. Then, bistatic results at grazing incidence

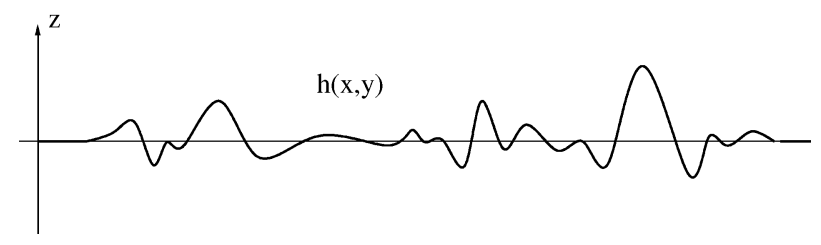

Fig. 1. Bounded perturbation of the plane.

are presented and compared in Section VI with two first order approximate methods, the small perturbation method and the small slope approximation, for rough surfaces with Gaussian pdf height and correlation function. Errors in the backscattering direction are outlined. The paper is finally concluded.

\section{BOUNDARY INTEGRAL FORMALISM}

In the right Cartesian coordinate $(\hat{\mathbf{x}}, \hat{\mathbf{y}}, \hat{\mathbf{z}})$ system with $z$-axis directed upward, the rough surface $\Sigma$ is a local perturbation of the $(x O y)$ plane with the vacuum as upper medium. The surface $\Sigma$ is given by a Cartesian equation $z=h(\mathbf{r})=h(x, y)$, and $\hat{\mathbf{n}}$ denotes its unit normal vector directed toward the vacuum (see Fig. 1).

Electric and magnetic fields are respectively denoted by $\mathbf{E}$ and $\mathbf{H}$, while superscripts $\mathbf{i}, \mathbf{r}, \mathbf{s}$ indicate respectively the incident, reflected and scattered field, and no superscript the total field. The reflected field is the field that would be reflected by the $(x O y)$ plane, so that the scattered field is defined in vacuum by $\mathbf{E}^{\mathbf{S}}=\mathbf{E}-\mathbf{E}^{\mathbf{i}}-\mathbf{E}^{\mathbf{r}}$. Let us notice that $\mathbf{E}^{\mathbf{S}}$ and the standard scattered field defined as $\mathbf{E}-\mathbf{E}^{\mathbf{i}}$ share the same incoherent scattering amplitudes. The magnetic fields are defined accordingly.

In order to obtain a boundary integral representation of the scattering problem, let us introduce the discontinuous vector functions $\mathbf{U}$ and $\mathbf{V}$

$$
\mathbf{U}=\left\{\begin{array}{l}
\mathbf{E}^{\mathbf{s}} \\
\mathbf{0}
\end{array} \quad \mathbf{V}= \begin{cases}\mathbf{H}^{\mathbf{s}} & \text { if } z>h(x, y) \\
\mathbf{0} & \text { if } z<h(x, y)\end{cases}\right.
$$

with jumps at the surface $[\hat{\mathbf{n}} \times \mathbf{U}]=\hat{\mathbf{n}} \times \mathbf{E}^{\mathbf{S}}=\mathbf{m}$ and $[\hat{\mathbf{n}} \times \mathbf{V}]=\hat{\mathbf{n}} \times \mathbf{H}^{\mathbf{s}}=\mathbf{j}$. Those equivalent surface currents are the unknowns of the boundary integral representation of the scattering problem. For an $\exp (-i \omega t)$ time dependence, the harmonic Maxwell equations for $\mathbf{U}$ and $\mathbf{V}$ write, in the sense of distributions

$$
\begin{aligned}
\operatorname{curl} \mathbf{U}-i \omega \mu_{0} \mathbf{V} & =\mathbf{m} \delta_{\Sigma} \\
\operatorname{curl} \mathbf{V}+i \omega \varepsilon_{0} \mathbf{U} & =\mathbf{j} \delta_{\Sigma}
\end{aligned}
$$

with $\delta_{\Sigma}$ the Dirac delta distribution associated with the surface. Those two equations can be combined to obtain a Helmholtz equation with right-hand side

$$
\Delta \mathbf{U}+K_{0}^{2} \mathbf{U}=-\operatorname{curl}\left(\mathbf{m} \delta_{\Sigma}\right)-i \omega \mu_{0} \mathbf{j} \delta_{\Sigma}+\operatorname{grad} \operatorname{div} \mathbf{U}
$$

$K_{0}=\omega \sqrt{\varepsilon_{0} \mu_{0}}$ denoting the wavenumber for vacuum. U satisfies an outgoing wave condition in both the upper and lower half spaces; we assume that this is a sufficient condition for (4) to have a unique solution, that writes as the convolution of the right-hand side and of the Green's function $G_{0}(\mathbf{R})=$ 
$-\left(\exp \left(i K_{0}|\mathbf{R}|\right) / 4 \pi|\mathbf{R}|\right)$. In order to discard the normal components, a supplementary curl operator is applied to (4). Finally, the function $\mathbf{V}$ can be expressed as

$$
\mathbf{V}=-\operatorname{curl}\left(G_{0} * \mathbf{j} \delta_{\Sigma}\right)+\frac{i}{\omega \mu_{0}} \operatorname{curl} \operatorname{curl}\left(G_{0} * \mathbf{m} \delta_{\Sigma}\right) .
$$

The same method applied to (3) gives

$$
\mathbf{U}=-\operatorname{curl}\left(G_{0} * \mathbf{m} \delta_{S}\right)-\frac{i}{\omega \varepsilon_{0}} \operatorname{curl} \operatorname{curl}\left(G_{0} * \mathbf{j} \delta_{S}\right) .
$$

To obtain integral equations, we must consider the limit of (5) and (6) when the observation point tends toward the surface. According to [23], the equations in vacuum are

$$
\begin{aligned}
& \left(\frac{1}{2}+M_{0}\right) \mathbf{j}-\frac{i}{\omega \mu_{0}} P_{0} \mathbf{m}=\mathbf{0} \\
& \left(\frac{1}{2}+M_{0}\right) \mathbf{m}+\frac{i}{\omega \varepsilon_{0}} P_{0} \mathbf{j}=\mathbf{0}
\end{aligned}
$$

with $M_{0}$ and $P_{0}$ the integral operators introduced by Martin and Ola in [24]. They write, for a tangential density $\mathbf{c}$ and two points $\mathbf{R}$ and $\mathbf{R}^{\prime}$ on the surface

$$
\begin{aligned}
M_{0} \mathbf{c}_{\mathbf{R}} & =\hat{\mathbf{n}} \times \operatorname{curl}_{\mathbf{R}} \int_{\Sigma} G_{0 \mathbf{R}, \mathbf{R}^{\prime}} \mathbf{c}_{\mathbf{R}^{\prime}} d S^{\prime} \\
P_{0} \mathbf{c}_{\mathbf{R}} & =\hat{\mathbf{n}} \times \operatorname{curl}_{\mathbf{R}} \operatorname{curl}_{\mathbf{R}} \int_{\Sigma} G_{0 \mathbf{R}, \mathbf{R}^{\prime}} \mathbf{c}_{\mathbf{R}^{\prime}} d S^{\prime} .
\end{aligned}
$$

Equations (7) and (8) are not independent. Therefore, except for the perfectly conducting case, for which $\mathbf{m}=-\hat{\mathbf{n}} \times$ $\left(\mathbf{E}^{\mathbf{i}}+\mathbf{E}^{\mathbf{r}}\right)$ is known, another independent relation between the unknowns $\mathbf{m}$ and $\mathbf{j}$ is required. In the frame of the impedance boundary condition, of which expression is $\hat{\mathbf{n}} \times \mathbf{E}=\hat{\mathbf{n}} \times Z[\hat{\mathbf{n}} \times$ $\mathbf{H}]$, this second relation writes

$$
\mathbf{m}-\hat{\mathbf{n}} \times Z \mathbf{j}=\hat{\mathbf{n}} \times \mathrm{Z}\left[\hat{\mathbf{n}} \times\left(\mathbf{H}^{\mathbf{i}}+\mathbf{H}^{\mathbf{r}}\right)\right]-\hat{\mathbf{n}} \times\left(\mathbf{E}^{\mathbf{i}}+\mathbf{E}^{\mathbf{r}}\right)
$$

with right-hand side vanishing outside the rough region, thus auguring a bounded right-hand side for the boundary integral equation. The perfectly conducting case corresponds to $Z=0$. The exact transmission problem with a homogeneous dielectric lower medium can be formalized by subtracting from the total field the field $\left(\mathbf{E}^{\mathrm{t}}, \mathbf{H}^{\mathrm{t}}\right)$ that would be transmitted in the dielectric through the $(x O y)$ plane. Associated integral equations can be written with right-hand sides that depend on $\hat{\mathbf{n}} \times\left(\mathbf{E}^{\mathbf{i}}+\mathbf{E}^{\mathbf{r}}-\right.$ $\left.\mathbf{E}^{\mathbf{t}}\right)$ and $\hat{\mathbf{n}} \times\left(\mathbf{H}^{\mathbf{i}}+\mathbf{H}^{\mathbf{r}}-\mathbf{H}^{\mathbf{t}}\right)$ which show bounded supports. Note that for lossy dielectrics, the use of $\left(\mathbf{E}^{\mathbf{t}}, \mathbf{H}^{\mathbf{t}}\right)$ that varies exponentially with $z$ might reveal tricky in a numerical method. For those materials, the integral relationship between the tangential components of the total fields will be benefitly approximated by a local impedance boundary condition.

\section{SCATTERED FIELD}

In the upper and lower half-spaces, $\mathbf{U}$ writes as a sum of outgoing plane waves

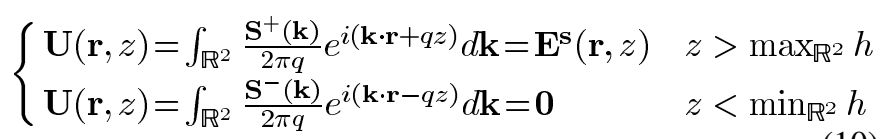

with $\mathbf{k}^{2}+q^{2}=K_{0}^{2}, \Re q \geq 0, \Im q \geq 0$ and, according to (6)

$$
\begin{array}{r}
\mathbf{S}^{ \pm}(\mathbf{k})=\frac{1}{4 \pi} \int_{\Sigma}\left(-\mathbf{K} \times \mathbf{m}_{\mathbf{R}^{\prime}}+\frac{1}{\omega \varepsilon_{0}} \mathbf{K} \times\left(\mathbf{K} \times \mathbf{j}_{\mathbf{R}^{\prime}}\right)\right) \\
\times \exp \left(-i \mathbf{K} \cdot \mathbf{R}^{\prime}\right) d S^{\prime}
\end{array}
$$

where $\mathbf{K}$ denotes the wavevector in vacuum $\mathbf{k} \pm q \hat{\mathbf{z}}$.

For an alpha-linearly polarized incident plane wave $\mathbf{E}^{\mathbf{i}}=$ $e^{i\left(\mathbf{k}_{\mathbf{0}} \cdot \mathbf{r}-q_{0} z\right)} \hat{\mathbf{p}}_{\boldsymbol{\alpha}}^{-}\left(\mathbf{k}_{\mathbf{0}}\right)$, the beta-polarized component of the upward scattering amplitude $S_{\beta \alpha}^{+}\left(\mathbf{k}, \mathbf{k}_{\mathbf{0}}\right)=\hat{\mathbf{p}}_{\boldsymbol{\beta}}^{+}(\mathbf{k}) \cdot \mathbf{S}_{\boldsymbol{\beta} \boldsymbol{\alpha}}^{+}(\mathbf{k})$ is related to the coefficient $S_{\beta \alpha}\left(\mathbf{k}, \mathbf{k}_{\mathbf{0}}\right)$ of the standard scattering matrix [25] by $S_{\beta \alpha}\left(\mathbf{k}, \mathbf{k}_{\mathbf{0}}\right)=S_{\beta \alpha}^{+}(\mathbf{k})+r_{\alpha} \delta\left(\mathbf{k}-\mathbf{k}_{\mathbf{0}}\right) \delta_{\beta \alpha}$, with $r_{\alpha}$ the relevant Fresnel reflection coefficient. As $S$ and $S^{+}$share the same incoherent part, the radar cross section writes $\sigma_{\beta \alpha}=$ $\left\langle\left|S_{\beta \alpha}^{+}-\left\langle S_{\beta \alpha}^{+}\right\rangle\right|^{2}\right\rangle$. Therefore, matrix $S_{\beta \alpha}^{+}\left(\mathbf{k}, \mathbf{k}_{\mathbf{0}}\right)$ for real values of $q$ and $q_{0}$ characterizes the far-field scattering from rough surface $\Sigma$, and its behavior at low-grazing incident $\left(q_{0} \rightarrow 0\right)$ and scattering $(q \rightarrow 0)$ angles is of particular interest here.

Theoretical limits at grazing have been studied by Tatarskii and Charnotskii in [22] for scalar waves scattered from rough surfaces, with Dirichlet and Neumann boundary conditions. Results for electromagnetic waves can be obtained with similar arguments; we just give outlines.

The behavior at grazing incidence is governed by the righthand side, and more precisely by $-\hat{\mathbf{n}} \times\left(\mathbf{E}^{\mathbf{i}}+\mathbf{E}^{\mathbf{r}}\right)$ for perfect conductors, $\hat{\mathbf{n}} \times \mathbf{Z} \hat{\mathbf{n}} \times\left(\mathbf{H}^{\mathbf{i}}+\mathbf{H}^{\mathbf{r}}\right)-\hat{\mathbf{n}} \times\left(\mathbf{E}^{\mathbf{i}}+\mathbf{E}^{\mathbf{r}}\right)$ in the impedance case and $\hat{\mathbf{n}} \times\left(\mathbf{E}^{\mathbf{i}}+\mathbf{E}^{\mathbf{r}}-\mathbf{E}^{\mathbf{t}}\right)$ and $\hat{\mathbf{n}} \times\left(\mathbf{H}^{\mathbf{i}}+\mathbf{H}^{\mathbf{r}}-\mathbf{H}^{\mathbf{t}}\right)$ in the transmission case. For example, it is easy to find from Fresnel coefficients that the last two vectors have all their components that tend to zero and behave as $q_{0}$ at grazing, whatever the polarization (denoted by $O\left(q_{0}\right)$ with the Landau notation). The same limit applies to the perfectly conducting case for horizontal $(\mathrm{H})$ polarization, defined by $\hat{\mathbf{p}}_{\mathbf{H}}^{ \pm}(\mathbf{k})=\hat{\mathbf{k}} \times \hat{\mathbf{z}}$, while it is only $O(1)$ in vertical $(\mathrm{V})$ polarization $\left(\hat{\mathbf{P}}_{\mathbf{V}}^{ \pm}(\mathbf{k})=(\mp q \hat{\mathbf{k}}+k \hat{\mathbf{z}}) / K\right)$. This behavior at grazing incidence angle is transmitted to unknowns $\mathbf{m}$ and $\mathbf{j}$ through integral equations and to the scattering amplitude through formula (11).

Since the scattering amplitude satisfies the reciprocity theorem [25], $S_{\beta \alpha}^{+}\left(\mathbf{k}, \mathbf{k}_{\mathbf{0}}\right)=(-)_{\beta \alpha} S_{\alpha \beta}^{+}\left(-\mathbf{k}_{\mathbf{0}},-\mathbf{k}\right)$ with $(-)_{\beta \neq \alpha}$ equals to -1 and $(-)_{\alpha \alpha}=+1$, the behavior at grazing scattering angles can be straightforwardly deduced, with main result that the whole scattering matrix is $O\left(q_{0} q\right)$ in the transmission case. The perfect conductor case is more complex because polarization dependent, as it appears on Table I. These last results are in agreement with [22], in the sense that the co-polarized $\mathrm{H}$ amplitude shares the same $O\left(q_{0} q\right)$ behavior with the Dirichlet problem, and the co-polarized $\mathrm{V}$ amplitude corresponds to the Neumann boundary condition. Note that the limit for the Neumann problem may depend on the exact configuration of the problem [26], [27].

In a numerical computation context, the correct behavior of the scattering amplitude around $q_{0} \rightarrow 0$ is naturally enforced. This comes directly from the fact that the excitation terms, and thus the right hand sides, actually decrease in amplitude with the correct speed at grazing. The answer is different when $q \rightarrow 0$, since the limit has been obtained through reciprocity. Here, we only address the cases where the limit at grazing scattering angles behave as $q$. Such a limit does not explicitly 
TABLE I

THE LOW-GRAZING BeHAVIOR OF THE SCATTERING AMPLITUdE, THE BistatiC and Monostatic Radar Cross SeCtions, Respectively, FOR Bounded PERTURbation of a Perfectly CONDUCting Plane MirRoR

\begin{tabular}{||c|c|c|c||}
\hline Polarization & $S_{\beta \alpha}^{+}\left(\mathbf{k}, \mathbf{k}_{\mathbf{0}}\right)$ & $\sigma_{\beta \alpha}\left(\mathbf{k}, \mathbf{k}_{\mathbf{0}}\right)$ & $\sigma_{\beta \alpha}(-\mathbf{k}, \mathbf{k})$ \\
\hline$\beta=\mathrm{H} \alpha=\mathrm{H}$ & $O\left(q_{0} q\right)$ & $O\left(q_{0}^{2} q^{2}\right)$ & $O\left(q^{4}\right)$ \\
$\beta=\mathrm{H} \alpha=\mathrm{V}$ & $O(q)$ & $O\left(q^{2}\right)$ & $O\left(q^{2}\right)$ \\
$\beta=\mathrm{V} \alpha=\mathrm{H}$ & $O\left(q_{0}\right)$ & $O\left(q_{0}^{2}\right)$ & $O\left(q^{2}\right)$ \\
$\beta=\mathrm{V} \alpha=\mathrm{V}$ & $O(1)$ & $O(1)$ & $O(1)$ \\
\hline
\end{tabular}

appear from formula (11). In addition, as recalled earlier, surface waves (SPP) may appear and the unknowns $\mathbf{m}$ and $\mathbf{j}$ may have a much larger support than the rough area. Therefore, with a bounded domain of study, the SPP is truncated and behaves as a sheet of current, radiating in both the lower and upper half-spaces through edge effects. Indeed, since the associated surface current is close to $\exp (i \mathbf{k} \cdot \mathbf{r})$, the scattering amplitude in the plane of propagation is, with good approximation, proportional to sinc $\left[\left(K_{0}-k\right) L / 2\right]$ ( $k$ and $L$ the wavenumber and the length of the rough area in the direction of propagation) which mainly contributes to grazing scattering angles and is an even function of $q$. Therefore, to get rid of these truncated SPP artifacts and enforce the theoretical behavior of the scattering amplitude at grazing scattering angles, we propose to compute the scattering amplitude through the combination

$$
\tilde{S}_{\beta \alpha}^{+}\left(\mathbf{k}, \mathbf{k}_{\mathbf{0}}\right)=S_{\beta \alpha}^{+}\left(\mathbf{k}, \mathbf{k}_{0}\right)-S_{\beta \alpha}^{-}\left(\mathbf{k}, \mathbf{k}_{\mathbf{0}}\right) .
$$

From (10), it is obvious that $S_{\beta \alpha}^{-}\left(\mathbf{k}, \mathbf{k}_{\mathbf{0}}\right)=0$ whatever $\mathbf{k}$, so combination (12) remains theoretically equal to $S_{\beta \alpha}^{+}$. It is to be noticed that, since (12) numerically enforces a $O(q)$ limit, it should not be used to compute the V-polarized component of the scattering amplitude in the perfectly conducting case, for any incident polarization.

\section{Numerical ImPlementation fOR PeRFECTLY CONDUCTING SURFACES}

Let us consider a rough surface, with roughness flattened except on a finite area. The flattening is mathematically realized by multiplying the elevation function by a Hanning function, as shown in Fig. 2. This ensures a smooth transition between the flat part and the rough part. The size of the transition is ruled by the parameters L1, L2, L1' and L2', the choice of these parameters depending on the rough surface under study. Fig. 3 shows the geometry of the tapered surface. The rectangle in dashed lines bounds the domain where $\hat{\mathbf{n}} \times\left(\mathbf{E}^{\mathbf{i}}+\mathbf{E}^{\mathbf{r}}\right)$ is non-zero. This domain is surrounded by plateaus of dimensions $\mathrm{P} 1, \mathrm{P} 2, \mathrm{P} 1^{\prime}$ and $\mathrm{P}^{\prime}$. Transitions and plateaus are sized to ensure that the right-hand side of (7) is bounded to the numerical domain of dimensions $2 \mathrm{~L}$ and $2 \mathrm{l}$, and represented in Fig. 3 by the rectangle in solid lines.

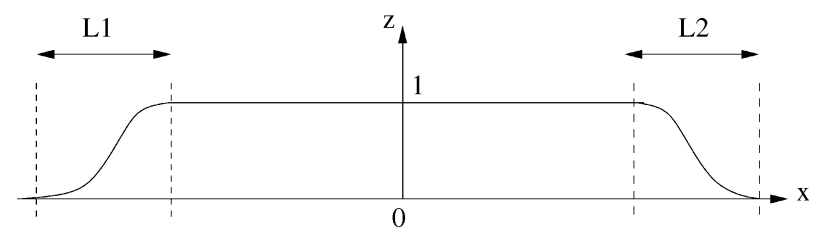

Fig. 2. The Hanning function.

Following Section II, the perfect conductor boundary condition writes $\mathbf{m}=-\hat{\mathbf{n}} \times\left(\mathbf{E}^{\mathbf{i}}+\mathbf{E}^{\mathbf{r}}\right)$. This can be inserted into (7) to get an integral equation

$$
\left(\frac{1}{2}+M_{0}\right) \mathbf{j}=-\frac{i}{\omega \mu_{0}} P_{0} \hat{\mathbf{n}} \times\left(\mathbf{E}^{\mathbf{i}}+\mathbf{E}^{\mathbf{r}}\right)
$$

with same kernel as the magnetic field integral equation (MFIE). As such, it can be solved by similar techniques. This equation, discretized by the method of moments (MoM), gives rise to a linear system $A X=B$ with a completely filled matrix $A$. This system can be solved iteratively at a $O\left(N^{2}\right)$ cost in memory and time, where $N$ is the number of surface unknowns. For two-dimensional surfaces, $N$ becomes very large and advanced numerical schemes have been proposed [6]. For instance, the sparse-matrix flat-surface approach [28] has a reduced memory cost of $O(N)$. This technique has been used in [29] with alternative iterative methods; the expression of the coefficients of the matrix $A$ appear in this paper. In [30], the method has been improved with a multilevel canonical grid technique; the time cost is now $O(N \log N)$ and details of the implementation can be found in [31].

Since the MFIE operator $M_{0}$ is weakly singular [23], [24], the method of moments can be applied with piecewise-constant basis functions and point matching. On the contrary, operator $P_{0}$ is hyper-singular, and the computation of the right-hand side of (13) is not trivial. With one differentiation of $P_{0}$ transferred onto the electric field, the right-hand side writes

$$
\begin{aligned}
- & \frac{i}{\omega \mu_{0}} P_{0} \hat{\mathbf{n}} \times\left(\mathbf{E}^{\mathbf{i}}+\mathbf{E}^{\mathbf{r}}\right)_{\mathbf{R}} \\
= & -\hat{\mathbf{n}} \times \int_{S}\left(i \omega \varepsilon_{0} G_{\mathbf{R}, \mathbf{R}^{\prime}} \hat{\mathbf{n}} \times\left(\mathbf{E}^{\mathbf{i}}+\mathbf{E}^{\mathbf{r}}\right)_{\mathbf{R}^{\prime}}\right. \\
& \left.\quad+\operatorname{grad}_{\mathbf{R}} G_{\mathbf{R}, \mathbf{R}^{\prime}} \hat{\mathbf{n}} \cdot\left(\mathbf{H}^{\mathbf{i}}+\mathbf{H}^{\mathbf{r}}\right)_{\mathbf{R}^{\prime}}\right) d S^{\prime} .
\end{aligned}
$$

For this integral to be computed numerically, $\hat{\mathbf{n}} \times\left(\mathbf{E}^{\mathbf{i}}+\mathbf{E}^{\mathbf{r}}\right)$ and $\hat{\mathbf{n}} \cdot\left(\mathbf{H}^{\mathbf{i}}+\mathbf{H}^{\mathbf{r}}\right)$ are expanded in piecewise-constant basis functions. The evaluation of the right-hand side vector $B$ of the linear system is obtained from two matrix-vector products, therefore requires $O\left(N^{2}\right)$ operations. With the multilevel canonical grid technique of [31], it is performed with reduced $O(N)$ memory and $O(N \log N)$ time requirements.

One should keep in mind that real materials have finite conductivity, thus that the transmission boundary condition should be addressed. In this case, all the components of the scattering amplitude behave as $O\left(q_{0} q\right)$ at grazing. Indeed, for low-grazing angles and vertical incident polarization, the Fresnel coefficients for finite and infinite conductivity show opposite limits (respectively -1 and +1 ) at grazing: the angle and conductivity limits cannot be commuted here. No real 


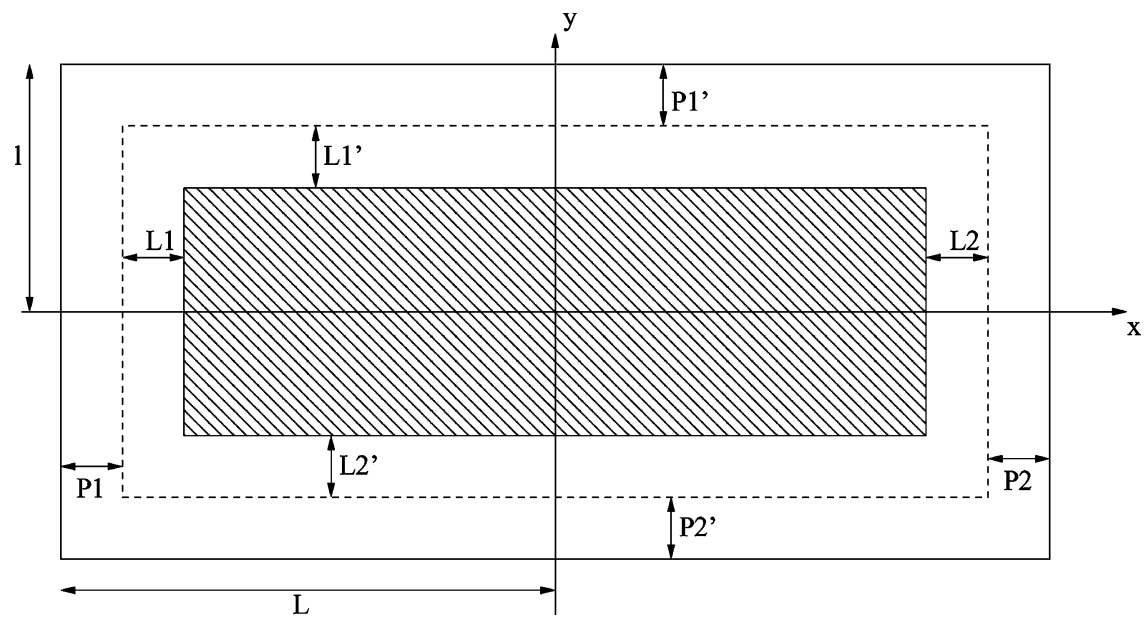

Fig. 3. Geometry of the tapered rough surface.

surface should be assumed perfectly conducting under vertically-polarized illumination around and beyond the Brewster angle. On the contrary, the conductivity has no effect on the horizontal Fresnel coefficient at grazing, and the perfectly conducting model is relevant. However, following Table I, only the co-polarized horizontal amplitude $S_{H H}^{+}$meets the correct behavior at grazing, and the cross-polarization should be considered unphysical. For the HH component of the scattering amplitude, the combination (12) as applied to (11) writes

$$
\begin{array}{r}
S_{H H}^{d i f}(\mathbf{k})=\frac{1}{2 \pi} \int_{\Sigma}\left\{i \sin (q h(\mathbf{r}))\left(k \hat{\mathbf{z}} \cdot \mathbf{m}+\omega \mu_{\mathbf{0}} \hat{\mathbf{h}} \cdot \mathbf{j}\right)\right. \\
+q \cos (q h(\mathbf{r})) \hat{\mathbf{k}} \cdot \mathbf{m}\} e^{-i \mathbf{k} \cdot \mathbf{r}} d S
\end{array}
$$

with obvious limit $O(q)$ at grazing, now.

In the two following sections, integral (13) is solved for the right-hand side (14) associated with a horizontally-polarized incident plane wave. The horizontal component of the scattering amplitude is computed from (15) and radar cross section is estimated by Monte Carlo average. The whole approach is called grazing MoM.

\section{VALIDATION AT NON-GRAZING ANGLES}

In this section, the grazing MoM is compared to the classical implementation of the MoM (denoted beam MoM thereafter), where the surface roughness is not bounded, but is enlightened by a tapered polarized beam with Gaussian envelope. Of course, only non-grazing incidence may be addressed here. The beam MoM has itself been validated by comparison with experimental data in [29], and has been used as a reference in numerous published works. All Monte Carlo averages have been performed on 200 samples.

The first studied surface has an isotropic Gaussian correlation function with height root mean square $h=0.083 \lambda$ and correlation radius $\ell=0.5 \lambda, \lambda$ denoting the electromagnetic wavelength. The incidence angle is $60^{\circ}$ from the normal. For the beam MoM, the rough surface is sampled at 8 points per wavelength and, in order to avoid edge effects, is set $64 \lambda$ long and $32 \lambda$ wide. The number of surface unknowns is thus $N=131072$.
For the grazing MoM, the surface area can be reduced, since the only requirement is that the dimensions of the rough part of the surface are much larger than the correlation radius. Here, the surface is square with sides $2 L=2 l=16 \lambda$, parameters $L 1=L 1^{\prime}=L 2=L 2^{\prime}=2 \lambda$ and plateaus of $1 \lambda$ (see Section IV and Fig. 3). With a sampling step of one eighth wavelength, the number of surface unknowns $N=16384$ is lowered by a factor eight, in comparison with the beam MoM.

Fig. 4 shows a comparison between the grazing MoM and the beam MoM co-polarized normalized radar cross section (NRCS), versus the scattering angle in the plane of incidence, for a non-grazing incidence of $60^{\circ}$. It is expected to find some discrepancy between the two curves in the region of the specularly reflected beam, from $50^{\circ}$ to $75^{\circ}$, as only incoherent scattering is considered for the grazing MoM, while it is total scattering for the beam MoM. As predicted by formula (15), the grazing MoM NRCS vanishes at forward and backward low-grazing angles, while the beam MoM NRCS, being estimated through a formula similar to (11), show non null limits. This explains discrepancies between the plots for angles lower than $-85^{\circ}$ or higher than $+85^{\circ}$. Outside these regions, the two methods coincide fairly well. One can also notice a slight vertical shift between the two curves over the whole diagram. This comes from the value of the area $A$ that normalizes the NRCS $\sigma^{0}=\sigma / A$ in the case of the grazing MoM, which should refer to the area of the rough part. This definition is however ambiguous, since the Hanning function makes a smooth transition between the plane and the roughness over distances set by parameters L1 and L2. Here, the normalizing area has been set to $A=(2 L-((L 1+L 2) / 2)-P 1-P 2)\left(2 l-\left(\left(L 1^{\prime}+\right.\right.\right.$ $\left.\left.\left.L 2^{\prime}\right) / 2\right)-P 1^{\prime}-P 2^{\prime}\right)$.

As a rigorous method, the grazing MoM predicts cross-polarization. In this case, formula (11) has to be used for computation of the scattering amplitude. For validation purpose, the $\mathrm{VH}$ component of the NRCS is plotted in the plane of incidence in Fig. 5 and compared to the beam MoM. The incident field of the grazing MoM is a perfectly horizontally-polarized plane wave. On the contrary, in the beam MoM, the surface is enlightened by a superposition of plane waves of which polarization cannot 


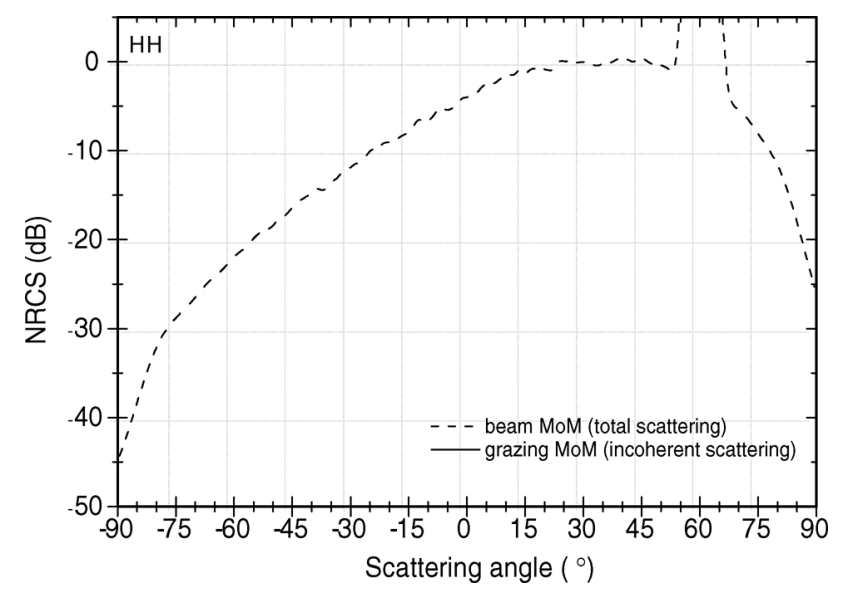

Fig. 4. Co-polarized component of the normalized scattering cross-section at $60^{\circ}$ incidence and Horizontal polarization versus scattering angle in the plane of incidence for the grazing MoM and the beam MoM. The surface is perfectly conducting with $0.083 \lambda$ height root mean square and an isotropic Gaussian correlation function of radius $0.5 \lambda$.

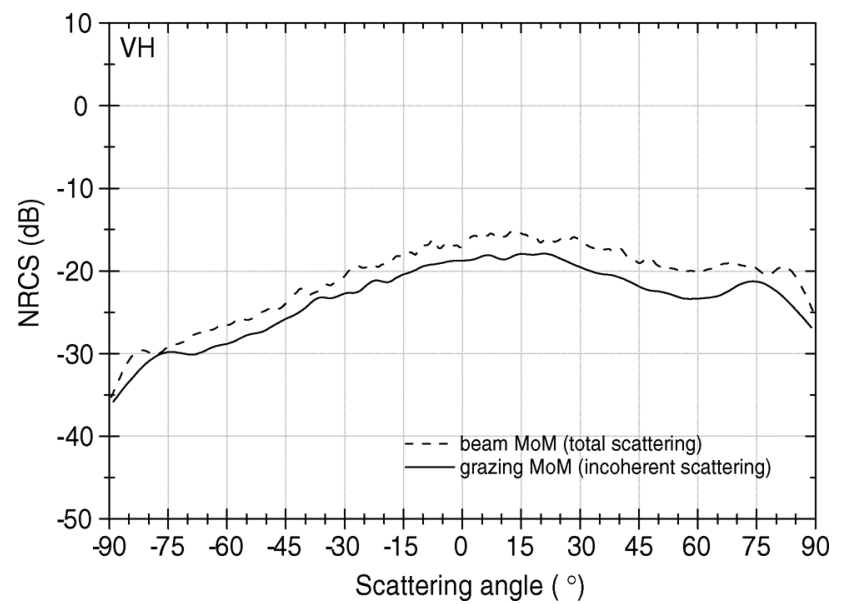

Fig. 5. Cross-polarized component of the normalized scattering cross-section at $60^{\circ}$ incidence and Horizontal polarization versus scattering angle in the plane of incidence for the grazing MoM and the beam MoM. The surface is perfectly conducting with $0.083 \lambda$ height root mean square and an isotrope Gaussian correlation function of radius $0.5 \lambda$.

be perpendicular to the plane of incidence [11]. Therefore, reflection of such a beam on a mere plane gives a field that comprises a non-zero vertically-polarized component. In the same way, the cross-polarized NRCS predicted by the beam MoM for a rough surface is overestimated. This explains the difference between the two curves of Fig. 5. To conclude this comparison, our opinion is that the grazing MoM is better suited than the beam MoM to characterize the cross-polarized response in the plane of incidence of a rough surface.

The grazing MoM can be applied to rougher surfaces. We now consider a second roughness with $0.25 \lambda$ height root mean square and $0.75 \lambda$ correlation radius, and a third one, with $0.50 \lambda$ height root mean square and $1.50 \lambda$ correlation radius, at $60^{\circ}$ incidence still. For these surfaces, the slope root mean square is 0.47 . The size of the surface samples for both methods is unchanged. For the second case (Fig. 6), the two models also fit very well. When the surface is very rough and its correlation length exceeds the wavelength, the two MoMs differ around

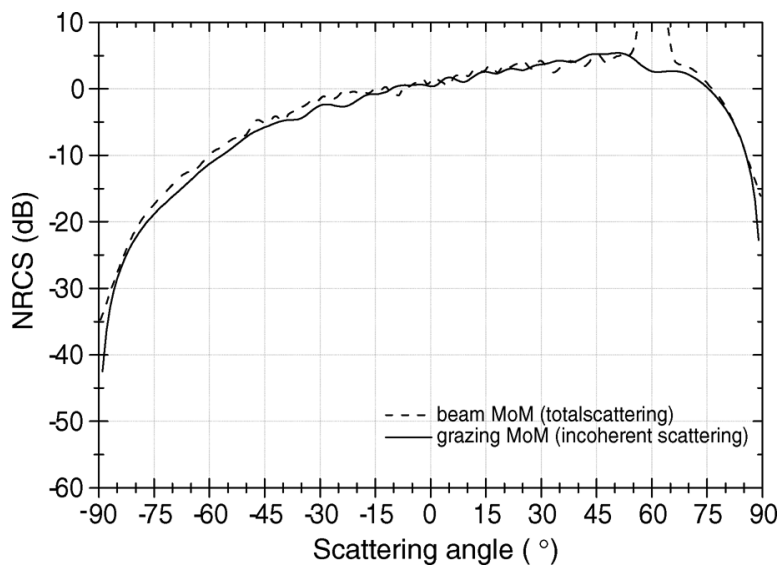

Fig. 6. Same as Fig. 4, with $0.25 \lambda$ height root mean square and $0.75 \lambda$ correlation radius.

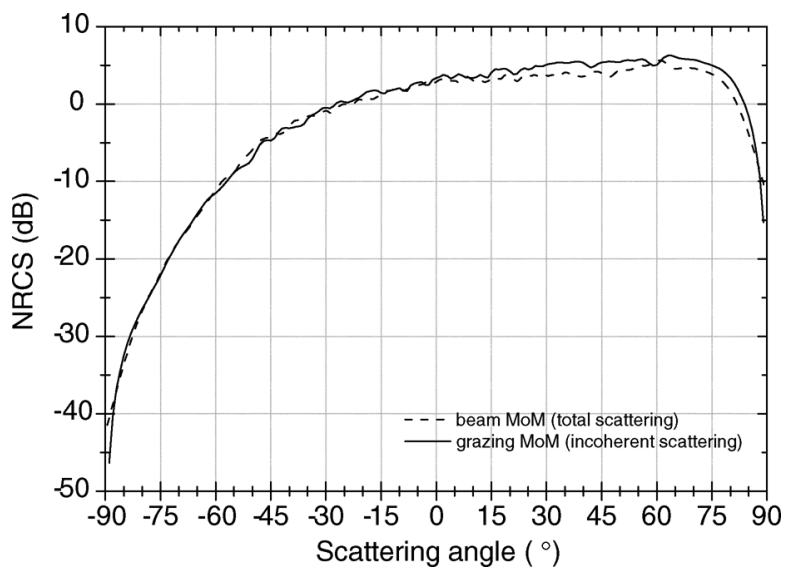

Fig. 7. Same as Fig. 4, with $0.50 \lambda$ height root mean square and $1.50 \lambda$ correlation radius.

the specular direction, as shown in Fig. 7. Here, the difference between the two representations of the problem-a tapered beam on an infinite roughness and a plane wave on a plane with bounded perturbation-shows off. However, comparison remains excellent for all backward angles, thus in backscattering.

These comparisons prove the validity and interest of the grazing MoM.

\section{COMPARISON With APPROXIMATE METHODS}

The grazing MoM, as a numerical solution of the rigorous scattering problem, can be used to check the validity of approximate models at low-grazing incidence, the backscattering direction being of particular interest for remote sensing applications. High-frequency asymptotics such as the Kirchhoff-tangent plane approximation or the Geometrical Optics, mainly suited for predicting scattering around the forward direction, will not be addressed here. We thus focus on the small perturbation method (SPM1) and the small slope approximation method (SSA1), both at first order. Note that more advanced and recently published methods such as [2] are claimed to handle lowgrazing, but we think that classical methods should be tested first. For Gaussian surfaces, SPM1 is usually given to be valid 


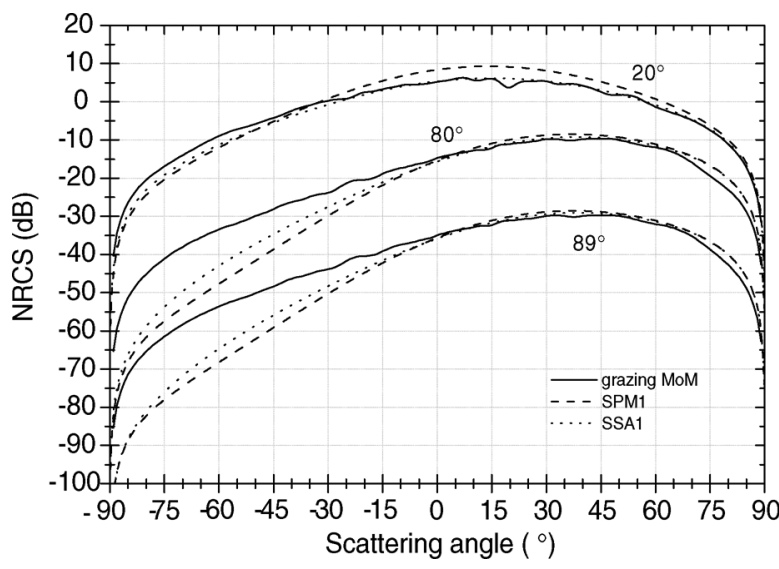

Fig. 8. Horizontal-horizontal component of the normalized scattering crosssection at $20^{\circ}, 80^{\circ}$ and $89^{\circ}$ incidence versus scattering angle in the plane of incidence for the grazing MoM, SPM1 and SSA1. The surface is perfectly conducting with $0.083 \lambda$ height root mean square and an isotropic Gaussian correlation of radius $0.5 \lambda$.

TABLE II

HORIZONTAL-HORIZONTAL COMPONENT OF THE BACKSCATTERING NRCS OF THE GRAZING MoM, SPM1 AND SSA1 IN dB FOR THREE VALUES OF THE Monostatic Angle in Degrees. The Surface is Perfectly Conducting WITH $0.083 \lambda$ HEIGHT RoOT MEAN SQUaRE AND AN ISOTROPIC GAUSSIAN CORRELATION OF RADIUS $0.5 \lambda$

\begin{tabular}{||c|c|c|c||}
\hline Monostatic angle & grazing MoM & SPM1 & SSA1 \\
\hline 20 & 2.48 & 4.10 & 1.87 \\
80 & -45.14 & -61.70 & -58.79 \\
89 & -85.44 & -102.87 & -102.82 \\
\hline
\end{tabular}

for height root mean square $a$ lower than $\lambda / 20$, while the criterion for SSA mixes $a$ with the slope root mean square $s$ : $K_{0} s a<1$. From the analytical expressions of the scattering amplitude, it appear that these two methods coincide when an exponential term can be linearized at first order, namely: $\exp [i(q+$ $\left.\left.q_{0}\right) h(\mathbf{r})\right] \simeq 1+i\left(q+q_{0}\right) h(\mathbf{r})$. Therefore, SPM1 and SSA1 should agree for low-grazing backscattering angles.

The study starts with a surface with a small roughness of $0.083 \lambda$ height root mean square and an isotropic Gaussian correlation of radius $0.5 \lambda$, already considered in the previous section. The height and the slope $\left(13.2^{\circ}\right.$ angle root mean square) are moderate, so SSA1 should behave well. Fig. 8 shows the comparison between these two approximations and the grazing MoM. Three angles are considered, namely $20^{\circ}, 80^{\circ} 89^{\circ}$. At $20^{\circ}$ incidence, SPM1 gives a correct indication of the shape of the scattering diagram, underestimating the NRCS between $-90^{\circ}$ and $-25^{\circ}$ and overestimating it beyond $-25^{\circ}$, with an error always smaller that $5 \mathrm{~dB}$. SSA1 is generally closer to the MoM, showing excellent accuracy on the major part of the diagram, from $-30^{\circ}$ to $+90^{\circ}$. However, outside this region, SSA1 underestimates the NRCS by several dB. When the angle of incidence increases, the gap between rigorous and approximate methods widens. SSA1 fits MoM on a region that goes tighter. As shown in Table II, SPM1 and SSA1 are very close to each other, but irrelevant for backscattering at low-grazing angles.

\section{CONCLUSION}

This paper presents a boundary integral method for the numerical solution of the rigorous problem of wave scattering from rough surfaces under grazing illumination. The model of a locally perturbated plane is adopted: a finite patch of rough surface has its roughness flattened at the edges. The boundary formulation unknowns are not the tangential components of the total field, but those of the scattered field, defined as the contribution from the rough area. This way, the numerical domain of study is correctly bounded, even if the incident field is a plane wave. Since no tapered beam is used, the sampled area is a priori independent of the incidence, and low-grazing angles are at hand. No supplementary assumption such as periodic boundary conditions or resistive loading is necessary.

This approach has been implemented on two-dimensional perfectly conducting surfaces, and validated by comparison with a reference numerical solution for surfaces with Gaussian correlation functions. Finally, the scattering diagrams predicted by the small perturbation method and the small slope approximation are compared to those given by this rigorous model, the grazing MoM, in the plane of incidence for incidence angles of $20^{\circ}, 80^{\circ}$ and $89^{\circ}$. One can see how the performances of these methods deteriorate drastically at backward scattering angles as the incidence goes to grazing, up to $17 \mathrm{~dB}$ in the backscattering direction at $89^{\circ}$ incidence for a surface with slope root mean square of 0.23 .

The same approach can be implemented to solve impedance or transmission problems. In such cases, hyper-singular integral operators cannot be avoided, and may require the use of more advanced discretization schemes, such as divergence-conforming basis functions.

This model is naturally aimed at investigating the numerous advanced approximate models that have appeared in the literature for the last decades [32], and sorting them in the context of low-grazing angles.

\section{REFERENCES}

[1] G. S. Brown, IEEE Trans. Antennas Propag. Special Issue on LowGrazing-Angle Backscattering from Rough Surfaces, vol. 46, pp. 1-2, 1998.

[2] A. Ishimaru, J. D. Rockway, and Y. Kuga, "Rough surface Green's function based on the first-order modified perturbation and smoothed diagram methods," Waves Random Media, vol. 10, pp. 17-31, 2000.

[3] A. Ishimaru, J. D. Rockway, Y. Kuga, and S.-W. Lee, "TE and TM Green's function for coherent and incoherent propagation over a finitely conducting rough surface," Radio Sci., vol. 37, pp. 1-13, 2002.

[4] D. Holliday, L. L. DeRaad, and G. J. St-Cyr, "Forward-backward: A new method for computing low-grazing angle scattering," IEEE Trans. Antennas Propag., vol. 44, pp. 722-729, 1996.

[5] D. A. Kapp and G. Brown, "A new numerical method for rough surface scattering calculations," IEEE Trans. Antennas Propag., vol. 44, pp. 711-721, 1996

[6] K. F. Warnick and W. C. Chew, "Numerical simulation methods for rough surface scattering," Waves in Random Media, vol. 11, no. 1, pp. 1-30, 2001.

[7] M. Saillard and A. Sentenac, "Rigorous solutions for electromagnetic scattering from rough surfaces," Waves Random Media, vol. 11, pp. R103-R137, 2001

[8] M. Saillard and D. Maystre, "Scattering from random rough surfaces: A beam simulation method," J. Optics, vol. 19, no. 4, pp. 173-176, 1988.

[9] E. I. Thorsos, "The validity of the Kirchhoff approximation for rough surface scattering using a gaussian roughness spectrum," J. Acoust. Soc. Am. A, vol. 82, pp. 78-92, 1989. 
[10] J. T. Johnson, "Surface currents induced on a dielectric half-space by a Gaussian beam: An extended validation for point matching method of moment codes," Radio Sci., vol. 32, pp. 923-934, 1997.

[11] H. Braunisch, Y. Zhang, C. O. Ao, S. E. Shih, Y. E. Yang, K. H. Ding, and J. A. Kong, "Tapered wave with dominant polarization stat for all angles of incidence," IEEE Trans. Antennas Propag., vol. 48, pp. 1086-1096, 2000.

[12] D. Maystre, "Electromagnetic scattering from perfectly conducting rough surfaces in the resonance region," IEEE Trans. Antennas Propag., vol. 31, pp. 885-895, 1983.

[13] J. V. Toporkov, R. S. Awadallah, and G. S. Brown, "Issues related to the uses of a Gaussian-like incident field for low-grazing-angle scattering," J. Opt. Soc. Am. A, vol. 16, pp. 176-186, Jan. 1999.

[14] J. T. Johnson, "A numerical study of low-grazing-angle backscatter from ocean-like impedance surfaces with the canonical grid method," IEEE Trans. Antennas Propag., vol. 46, pp. 114-120, 1998.

[15] D. E. Barrick, "Near-grazing illumination and shadowing of rough surfaces," Radio Sci., vol. 30, pp. 563-580, May 1995.

[16] R. Chen and J. C. West, "Analysis of scattering from rough surfaces at large incidence angles using a periodic-surface moment method," IEEE Trans. Geophys. Remote Sensing, vol. 33, no. 5, pp. 1206-1213, Sep. 1995.

[17] T. Senior and V. Liepa, "Backscattering from tapered resistive strips," IEEE Trans. Antennas Propag. [Legacy, pre-1988], vol. 32, no. 7, pp. 747-751, 1984.

[18] R. Haupt and V. Liepa, "Synthesis of tapered resistive strips," IEEE Trans. Antennas Propag. [Legacy, pre-1988], vol. 35, no. 11, pp. $1217-1225,1987$.

[19] Z. Zhao, L. Li, J. Smith, and L. Carin, "Analysis of scattering from very large three-dimensional rough surfaces using MLFMM and ray-based analyses," IEEE Antennas Propag. Mag., vol. 47, no. 3, pp. 20-30, 2005.

[20] J. C. West, "On the control of edge diffraction in numerical rough surface scattering using resistive tapering," IEEE Trans. Antennas Propag., vol. 51, no. 11, pp. 3180-3183, 2003.

[21] A. Meier and S. N. Chandler-Wilde, "On the stability and convergence of the finite section method for integral equation formulations of rough surface scattering," Math. Methods Appl. Sci., vol. 24, no. 4, pp. 209-232, 2001.

[22] V. I. Tartarskii and M. Charnotskii, "On the universal Behavior of scattering from a rough surface for small grazing angles," IEEE Trans. Antennas Propag., vol. 46, pp. 67-72, 1998.

[23] D. Colton and R. Kress, Integral Equations in Scattering Theory. New York: Wiley-Interscience, 1983.

[24] P. A. Martin and P. Ola, "Boundary integral equations for the scattering of electromagnetic waves by a homogeneous dielectric obstacle," Proc. Roy. Soc. Edinburgh, vol. 123A, pp. 185-208, 1993.

[25] A. G. Voronovich, Wave Scattering From Rough Surfaces. Berlin: Springer-Verlag, 1994.

[26] D. E. Barrick, "Grazing behavior of scatter and propagation above any rough surface," IEEE Trans. Antennas Propag. , vol. 46, pp. 73-83, 1998.

[27] I. M. Fuks, V. I. Tartarskii, and M. Charnotskii, "Behavior of scattering from a rough surface at small grazing angles," Waves Random Media, vol. 9, pp. 295-305, 1999.

[28] K. Pak, L. Tsang, C. H. Chan, and J. T. Johnson, "Backscattering enhancement of electromagnetic waves from two-dimensional perfectly conducting random rough surfaces based on Monte Carlo simulations," J. Opt. Soc. Amer. A, vol. 12, pp. 1491-1499, 1995.
[29] G. Soriano and M. Saillard, "Scattering of electromagnetic waves from two-dimensional rough surfaces with impedance approximation," $J$. Opt. Soc. Amer. A, vol. 18, no. 1, pp. 124-133, 2001.

[30] S. Q. Li, C. H. Chan, M. Y. Xia, B. Zhang, and L. Tsang, "Multilevel expansion of the sparse-matrix canonical grid method for two-dimensional random rough surfaces," IEEE Trans. Antennas Propag., vol. 47, no. 4, pp. 752-763, April 2001

[31] G. Soriano, C.-A. Guérin, and M. Saillard, "Scattering by two-dimensional rough surfaces: Comparison between the method of moments, kirchhoff and small-slope approximations," Waves Random Media, vol. 12, pp. 63-83, 2002.

[32] T. Elfouhaily and C. A. Guérin, "A critical survey of approximate scattering wave theories from random rough surfaces," Waves Random Media, vol. 14, pp. R1-R40, 2004.

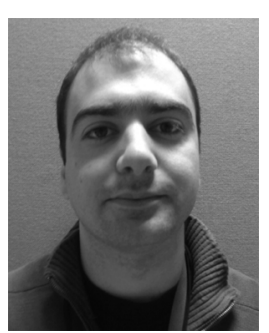

Philippe Spiga was born in Draguignan, France, in 1980. He received the M.S. degree in physics from the Paul Cezanne University, Marseille, France, in 1996. He is currently working toward the Ph.D. degree at DCNS, Toulon, France.

$\mathrm{He}$ is also on the Electromagnetic Compatibility Team collaborating with the Laboratoire de sondage electromagnetiques de l'environnement terrestre [LSEET] at the Fresnel Institute, Marseille. He works on the surface scattering at grazing angle.

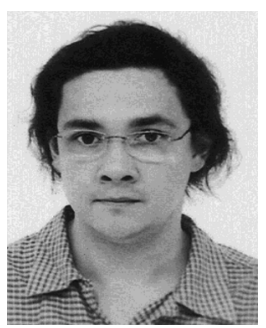

Gabriel Soriano was born in Paris, France, in 1972. He received the M.S. and Ph.D. degrees in physics from Paul Cezanne University, Marseille, France, in 1996 and 2000, respectively.

He is currently an Associate Professor at the Paul Cezanne University and is also a member of the ElectroMagnetical and Optical remote Sensing (SEMO) team at the Fresnel Institute, Marseille. He works on wave scattering from rough surfaces and associated numerical methods.

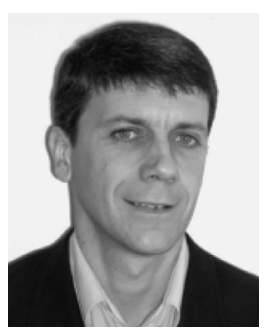

Marc Saillard was born in Marseille, France, in 1961. He received the "Agrégation" degree in physics, in 1985, and the Ph.D. degree in 1990 from the University of Marseille, France.

From 1990 to 1997, he was an Assistant Researcher at the Electromagnetic Optics Laboratory, National Center for Scientific Research (CNRS). In 1997, he joined the University of Marseille as a Professor where he ran the Remote Sensing Group of the Fresnel Institute. In 2003, he moved to the University of Toulon, Toulon, France, as a Professor, where he joined the Laboratoire de sondage electromagnetiques de l'environnement terrestre (LSEET). His professional interests lie in surface and volume scattering, inverse scattering, and remote sensing. 